\title{
A Case of AL Amyloidosis with Renal Rupture
}

\author{
Yuko Iwabuchi ${ }^{1}$, Mitsuyo Itabashi ${ }^{1}$, Tetsuri Yamashita ${ }^{1}$, Kunio Kawanishi ${ }^{1}$, Chiari Kojima ${ }^{1}$, \\ Takashi Takei ${ }^{1}$, Junko Arai ${ }^{1}$, Kazuho Honda ${ }^{2}$ and Kosaku Nitta ${ }^{1}$
}

\begin{abstract}
We report a 59-year-old woman with AL amyloidosis who presented with massive bleeding from the right kidney, in whom emergency surgery proved to be life saving. The patient had been diagnosed as having AL amyloidosis 16 years previously. After 5 years, hemodialysis had been initiated. In 2007, a large right-sided perinephric, intracapsular hematoma was detected. Right nephrectomy was performed and the patient recovered with no sequelae. Histopathological examination revealed a greater degree of amyloid deposition in the resected kidney than that at the time of diagnosis. Amyloid angiopathy may promote bleeding.
\end{abstract}

Key words: AL amyloidosis, renal rupture, hemodialysis, long-term survivor

(Inter Med 49: 2129-2132, 2010)

(DOI: 10.2169/internalmedicine.49.3029)

\section{Introduction}

AL amyloidosis is reportedly associated with a very poor prognosis $(1,2)$. Kyle and Gertz et al, based on their study of 474 patients with AL amyloidosis, reported a median survival of 13.2 months, with about half presenting with renal failure at diagnosis (2). Gertz and Kyle reported a median survival time of patients with primary amyloidosis of 20.4 months, with a 5-year survival rate of $19.6 \%$ (3). In addition, AL amyloidosis is known to be associated with potentially life-threatening hemorrhage because of the frequent occurrence of acquired hemostatic abnormalities, including coagulation factor deficiencies, hyperfibrinolysis, and platelet dysfunction (4). Cerebral bleeding, gastrointestinal bleeding, and bleeding after diagnostic procedures, such as organ biopsy, are the most frequently encountered bleeding manifestations (5-7). On the other hand, there are few reports of AL amyloidosis complicated by renal rupture. This is the first report of a patient with $\mathrm{AL}$ amyloidosis complicated with renal rupture who remains alive 16 years after the initial diagnosis.

\section{Case Report}

The patient, a 43-year-old physically active Japanese woman, became aware of edema of the lower extremities in May 1992. She had no significant past medical history.

In May 1993, she was admitted to our Nephrology Department for evaluation of the edema. On admission, she was $153 \mathrm{~cm}$ in height and weighed $59.0 \mathrm{~kg}$. Her body temperature was $37.0^{\circ} \mathrm{C}$, blood pressure $110 / 72 \mathrm{mmHg}$ and pulse rate 78/min. M-bows reacting with both anti-IgG and anti- $\lambda$ antibodies were detected in serum and urine. There were no abnormalities on chest X-ray or electrocardiography. An echocardiogram showed ventricular septal and left ventricular free wall thickening, with a normal ejection fraction. A bone marrow aspirate showed normocellularity, with a $3.6 \%$ of plasma cells, without dysplasia. A diagnosis of nephrotic syndrome was made, followed by percutaneous renal biopsy. Histological examination revealed no evidence of glomerular obsolescence or crescent formation. There was no evidence of tubular atrophy inflammation with interstitial infiltrates. Polarized light microcopy of biopsy specimens stained with Congo red revealed mild amyloid deposition in the mesangium and under the pericapillary wall (Fig. 1). In addition, the deposits were still detectable after treatment of the specimens with $\mathrm{KMnO}_{4}$, suggesting the diagnosis of $\mathrm{AL}$ amyloidosis. In addition to the renal tissues, abdominal fat tissue was also positive for AL amyloid deposits. There was no evidence of other organ deposit of amyloid protein. The patient was hospitalized and started on MP therapy. Subse-

${ }^{1}$ Department of Medicine, Kidney Center, Tokyo Women's Medical University, Tokyo and ${ }^{2}$ Department of Pathology II, Tokyo Women's Medical University, Tokyo

Received for publication October 16, 2009; Accepted for publication May 28, 2010

Correspondence to Dr. Mitsuyo Itabashi, m-itaba@ fb4.so-net.ne.jp 

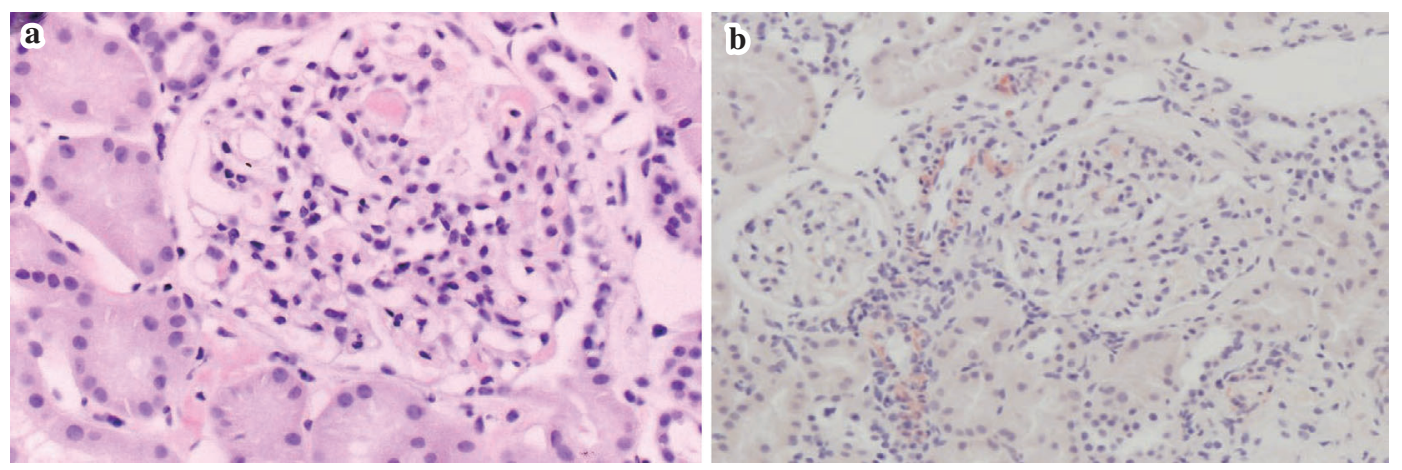

Figure 1. Renal biopsy sections with Hematoxylin and Eosin staining (a) and Congo-red staining (b) are shown. In the glomerulus, amyloid deposition in the capillary wall was mild.
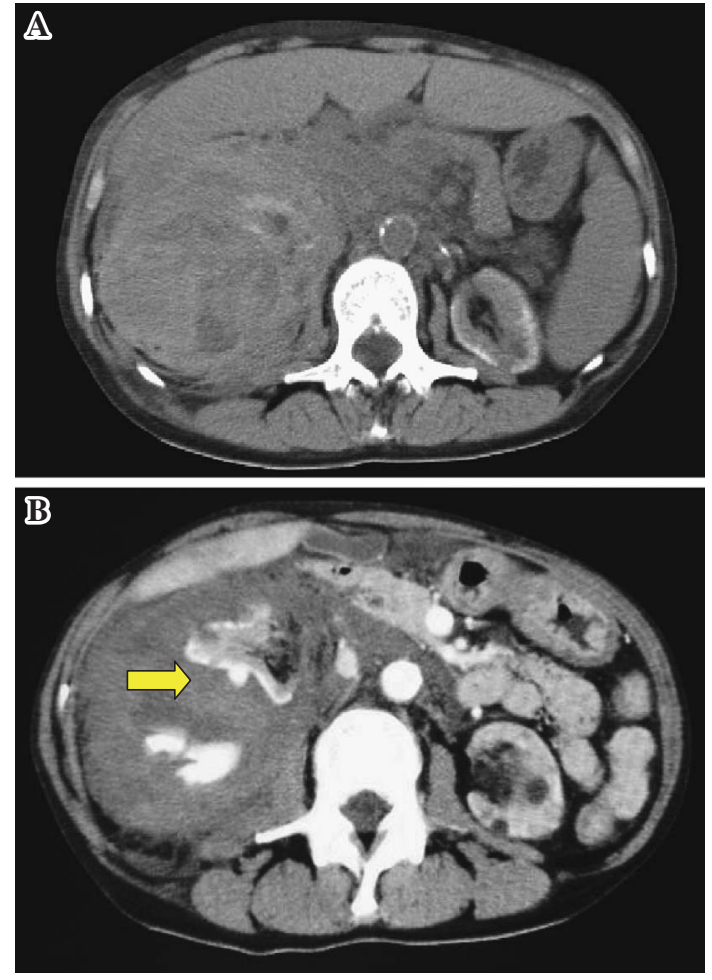

Figure 2. CT revealed a large right-sided perinephric, intracapsular hematoma. A: plain CT, B: early phase of contrast CT

quently, she was maintained on oral prednisolone on an outpatient basis, with the daily dose tapered by $10 \mathrm{mg}$ every 4 weeks. Following this treatment, M-bows became undetectable, and serum IgG decreased to $687 \mathrm{mg} / \mathrm{mL}$. However, urinary protein leakage, including of immunoglobulin, was still detectable at 4-5 g/day. The patient was therefore maintained on prednisolone at the daily dose of $5 \mathrm{mg}$, since there was no evidence of any increase in serum immunoglobulin levels. BUN and serum creatinine levels then began to increase gradually. In September 1998, the patient was admitted and hemodialysis was initiated because of progressive renal dysfunction. An abdominal echography showed that both kidneys were of normal size and shape with several cysts. Serum monoclonal immunoglobulin was slowly increased during maintenance dialysis. However, no extra- medullary tumor was detected. We did not perform more aggressive therapy due to fear of infection, because her IgG levels were low. In mid-January 2007, the patient complained of a sharp pain of sudden onset on the right side of her abdomen, and was found to have tachycardia and hypotension. Her hemoglobin dropped from $10.5 \mathrm{~g} / \mathrm{dL}$ to 7.3 $\mathrm{g} / \mathrm{dL}$ within a few hours, although no evidence of hemorrhage was detectable. Clinical parameters regarding homeostasis, such as platelet, fibrinolysis and coagulation were normal. Abdominal CT revealed a large right-sided perinephric, intracapsular hematoma (Fig. 2). Although no active bleeding was present, the severe serum hemoglobin drop and large size of the right-sided perinephric, intracapsular hematoma suggested the possibility of renal artery rupture. Right nephrectomy was thus performed and the patient recovered without sequelae. According to the examination of the removed kidney, the bleeding site was identified as the right renal artery in renal hilum. Histological findings did not reveal microaneurysm. There were many small cysts sized about $5 \mathrm{~mm}$, but there was no evidence of bleeding from cysts. Polarized light microscopic examination of resected kidney specimens stained with Dylon revealed dense amyloid deposits in all glomeruli and vessels (Fig. 3). Staining of the specimens for $\beta 2$-microglobulin was negative.

After right nephrectomy, the patient remained stable on regular hemodialysis, and low-dose oral prednisolone. We assessed other organ involvement of amyloid deposition. She had no symptoms of neuropathy or diarrhea, but amyloid deposition was positive in rectum biopsy. Thorough echocardiography was performed, and her calculated ejection fraction was 58\% with ventricular septal thickness which is strongly suggestive of cardiac amyloidosis.

\section{Discussion}

$\mathrm{AL}$ amyloidosis is known to be associated with a poor prognosis. Despite several attempts at the application of new therapeutic strategies, including bone marrow transplantation and myeloablative chemotherapy, no obvious survival improvement has been achieved during the past 3 decades. A series of previous reports has shown that the median survival duration of patients with primary amyloidosis and sec- 

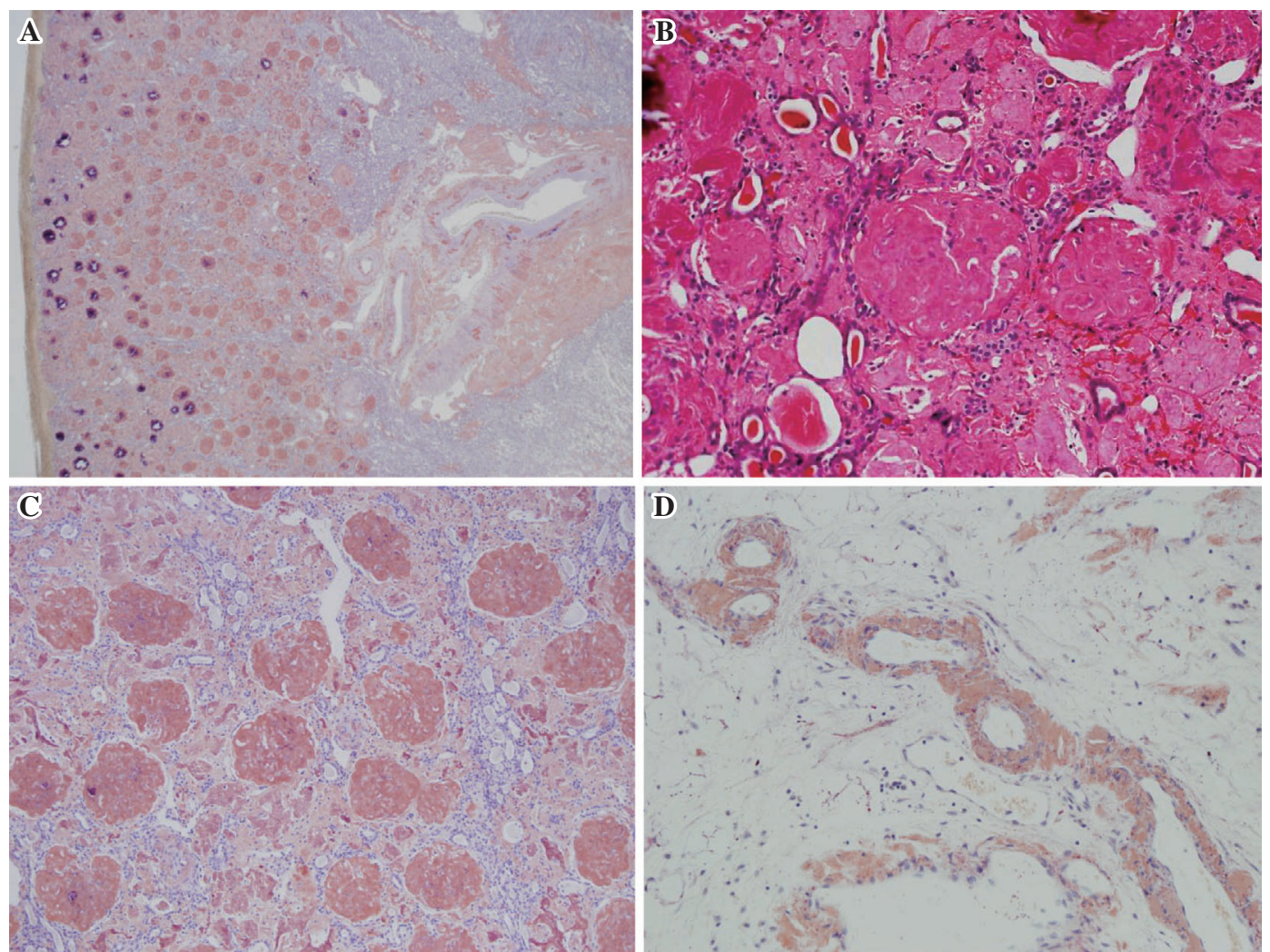

Figure 3. Right kidney specimen with Dylon (A, C, D) and Hematoxylin and Eosin (B) staining is shown. Amyloid depositions were positive in the glomerulus and perivascular area (A: $\times 40, B$ : $\times 200)$. Advanced amyloid deposition with obliteration of glomerular capillary tufts $(\mathrm{C}: \times 100)$. Severe amyloid deposition in blood vessels $(\mathrm{D}: \times 400)$.

ondary amyloidosis is no longer than 5 to 14 months (1-3). Some reports have indicated that no patient with primary amyloidosis has survived longer than 12 months after the diagnosis (8-11). The prognosis becomes even worse when patients develop complications such as renal insufficiency and heart failure $(1,2)$. Kyle et al (12) reported, based on the results of a multivariate analysis of $168 \mathrm{AL}$ amyloidosis patients, that renal function was a predictor of survival at 12 months after the diagnosis. It has also been reported that in most cases of AL amyloidosis with nephrotic syndrome, the nephrotic syndrome progresses to renal insufficiency, necessitating initiation of hemodialysis, and multiorgan failure, which directly adversely affects prognosis (13). The present case was a patient who showed long-term survival despite developing complicating nephrotic syndrome and renal dysfunction.

It has been reported that AL amyloidosis can be associated with life-threatening hemorrhage (4), because of the frequent occurrence in these patients of coagulation factor deficiencies, hyperfibrinolysis, and platelet dysfunction. Some patients with AL amyloidosis have been reported to show an acquired coagulation disorder characterized by factor $\mathrm{X}$ deficiency, considered to be due to rapid immobilization of circulating factor X (14-17). The present patient had no coagulation dysfunction, and no association with factor $\mathrm{X}$ deficiency, prior to the detection of the hematoma. Bleeding in this setting may be promoted by the occurrence of amyloid angiopathy, with increased fragility of blood vessels and impaired vasoconstriction.

The present patient showed massive amyloid deposition in the glomeruli and small vessels. In addition, massive amyloid deposition in the medium-to-large vessels, which caused the large hematoma with bleeding, was also observed. However, there was no microaneurysm formation or double barreling (18). Interestingly, our patient showed a long survival in response to MP therapy. However, a significantly greater degree of amyloid deposition was found in the surgically resected kidney specimen than that found at the time of diagnosis. Thus, our maintenance therapy was probably ineffective for controlling the disease activity, although it did successfully prevent complicating infections.

We report the first case of a patient with AL amyloidosis with renal rupture who remains alive 16 years after the initial diagnosis.

\section{References}

1. Kyle RA, Gertz MA, Greipp PR, et al. Long-term survival (10 years or more) in 30 patients with primary amyloidosis. Blood $\mathbf{9 3}$ : 1062-1066, 1999.

2. Kyle RA, Gertz MA. Primary systemic amyloidosis: clinical and laboratory features in 474 cases. Semin Hematol 32: 45-59, 1995.

3. Gertz MA, Kyle RA. Primary systemic amyloidosis - a diagnostic primer. Mayo Clin Proc 64: 1505-1519, 1989.

4. Sucker C, Hetzel GR, Grabensee B, Stockschlaeder M, Scharf RE. Amyloidosis and bleeding: pathophysiology, diagnosis, and ther- 
apy. Am J Kidney Dis 47: 947-955, 2006.

5. Revesz T, Ghiso J, Lashley $\mathrm{T}$, et al. Cerebral amyloid angiopathies: a pathologic biochemical, and genetic view. J Neuropathol Exp Neurol 62: 885-898, 2003.

6. Usui M, Matsuda S, Suzuki H, Hirata K, Ogura Y, Shiraishi T. Gastric amyloidosis with massive bleeding requiring emergency surgery. J Gastroenterol 35: 924-928, 2000.

7. Eiro M, Katoh T, Watanabe T. Risk factors for bleeding complications in percutaneous renal biopsy. Clin Exp Nephrol 9: 40-45, 2005.

8. Daniels JD, Hewlett JS. Renal manifestations in multiple myeloma and in primary amyloidosis. Cleve Clin Q 37: 181-187, 1970.

9. Pick AI, Frohlichmann R, Lavie G, Duczyminer M, Skvaril F. Clinical and immunochemical studies of 20 patients with amyloidosis and plasma cell dyscrasia. Acta Haematol 66: 154-167, 1981.

10. Alexanian R, Fraschini G, Smith L. Amyloidosis in multiple myeloma or without apparent cause. Arch Intern Med 144: 21582160, 1984.

11. Pruzanski W, Katz A. Clinical and laboratory findings in primary generalized and multiple-myeloma-related amyloidosis. Can Med Assoc J 114: 906-909, 1976.

12. Kyle RA, Greipp PR, O'Fallon WM. Primary systemic amyloidosis: multivariate analysis for prognostic factors in 168 cases.
Blood 68: 220-224, 1986.

13. Montseny JJ, Kleinknecht D, Meyrier A, et al. Long-term outcome according to renal histological lesions in 118 patients with monoclonal gammopathies. Nephrol Dial Transplant 13: 1438-1445, 1998.

14. Furie B, Voo L, McAdam KP, Furie BC. Mechanism of factor $X$ deficiency in systemic amyloidosis. N Engl J Med 304: 827-830, 1981.

15. Marcatti M, Mauri S, Tresoldi M, et al. Unusual bleeding manifestations in a case of primary amyloidosis with factor $\mathrm{X}$ deficiency but elevations of in vivo markers of thrombin formation and activity. Thromb Res 80: 333-337, 1995.

16. Mumford AD, O’Donnell J, Gillmore JD, Manning RA, Hawkins PN, Laffan M. Bleeding symptoms and coagulation abnormalities in 337 patients with AL-amyloidosis. Br J Haematol 110: 454460, 2000.

17. Enjeti AK, Walsh M, Seldon M. Spontaneous major bleeding in acquired factor $\mathrm{X}$ deficiency secondary to AL-amyloidosis. Haemophilia 11: 535-538, 2005.

18. Maruyama K, Ikeda S, Ishihara T, Allsop D, Yanagisawa N. Immunohistochemical characterization of cerebrovascular amyloid in 46 autopsied cases using antibodies to beta protein and cystatin C. Stroke 21: 397-403, 1990.

(C) 2010 The Japanese Society of Internal Medicine http://www.naika.or.jp/imindex.html 